Recherches amérindiennes au Québec

\title{
Formation à la vidéo et développement de médias audiovisuels pour les autochtones du Québec
} Quel rôle pour le Wapikoni mobile?

Video Training and the Development of Broadcasting for First

Nations in Québec

What is the Role of Wapikoni Mobile?

Formación en video y desarrollo de los medios de comunicación audiovisuales para los indígenas de la provincia de Quebec ¿Qué papel debe jugar el Wapikoni Mobile?

\section{Antonin Serpereau}

Volume 42, numéro 1, 2012

Autochtones et médias

URI : https://id.erudit.org/iderudit/1023719ar

DOI : https://doi.org/10.7202/1023719ar

Aller au sommaire du numéro

Éditeur(s)

Recherches amérindiennes au Québec

ISSN

0318-4137 (imprimé)

1923-5151 (numérique)

Découvrir la revue

Citer cet article

Serpereau, A. (2012). Formation à la vidéo et développement de médias audiovisuels pour les autochtones du Québec : quel rôle pour le Wapikoni mobile ? Recherches amérindiennes au Québec, 42(1), 41-48.

https://doi.org/10.7202/1023719ar

\section{Résumé de l'article}

En quoi un projet d'intervention par la formation à la vidéo peut-il contribuer à l'émergence de médias autochtones ? C'est cette question qui est posée dans le présent article à travers le cas du Wapikoni mobile, organisme qui met sur pied des ateliers de formation vidéo dans des communautés autochtones du Québec depuis 2004. De manière exploratoire, l'auteur analyse l'histoire des médias audiovisuels publics autochtones du Grand Nord canadien pour voir dans quelle mesure ce projet peut participer au renforcement du paysage médiatique autochtone au Québec, notamment par rapport à la perspective de constitution de contre-publics subalternes. 


\section{Antonin Serpereau}

Centre de

recherche GRICIS

et Université du

Québec à Montréal

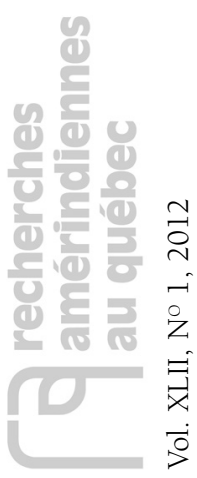

D URANT L'ÉTÉ 1966, deux anthropologues américains, Sol Worth et John Adair, organisent une formation audiovisuelle dans une communauté navajo. Ils entendent constituer un corpus de productions audiovisuelles réalisées entièrement par des Navajos, afin de voir si les résultats diffèrent des standards de l'industrie en termes de narration et, incidemment, mettre au jour, espèrentils, des structures à même de les renseigner sur un système de pensée autochtone (Worth et Adair 1997). La motivation centrale de ce projet est scientifique, peut-être dans le mauvais sens du terme : acteurs certainement, les participants navajos restent confinés au rang d'objets d'étude. Lintroduction d'une nouvelle technologie médiatique n'est pas ancrée dans un quelconque projet d'émancipation, ni même simplement envisagée comme ayant une quelconque utilité pour cette communauté. Comme le remarque Faye Ginsburg, ce premier transfert de techniques audiovisuelles à des autochtones reste, au fond, stérile et paternaliste (Ginsburg 1995 : 262). Fort heureusement, d'autres dynamiques ont, depuis, vu le jour, et l'histoire des formations audiovisuelles réservées à des populations autochtones montre que la préoccupation autour de la pertinence de ces techniques pour ces populations est devenue centrale (Michaels 1994; Gallois et Carelli 1995; Ginsburg 1995; Rodriguez 2001; Turner 1990). « Vidéo dans les villages (Video nas Aldeias) » est l'un des projets les plus connus; il concerne les Kayapos, au Brésil (la campagne de sensibilisation contre la déforestation de l'Amazonie, en 1989, et l'image du chef Ropni en compagnie du chanteur Sting n'y sont sans doute pas étrangères). Son évolution est éclairante. Au milieu des années quatre-vingt, deux anthropologues et documentaristes, Monica Feitosa et Renato Pereira, ont enseigné le maniement d'une caméra vidéo à deux jeunes Kayapos de la communauté de Gorotire (Mato Grosso, Brésil). En 1990, l'anthropologue Terence Turner, répondant à une demande de la part de certains chefs kayapos, entreprend un travail de formation en s'appuyant sur ces fondations. Confronté à un manque de ressources matérielles et de connaissances pour le montage, il prend appui sur le Centre de travail indigène (Centro de Trabalho Indigenista, CTI). Vincent Carelli, documentariste formé en anthropologie, se charge de cette formation au montage. Cette rencontre est essentielle, car elle provoque une modification du propre projet de Carelli, Vidéos dans les villages. Ce dernier, lancé en 1987 au sein du CTI, était jusqu'alors envisagé comme une offre de service audiovisuel auprès des communautés autochtones. À la suite de cette collaboration, une dynamique de formation, c'est-à-dire de transfert technologique, s'est progressivement structurée autour d'une 
logique de formation à même de permettre aux Kayapos de produire leurs propres contenus audiovisuels (Turner 1990; Gallois et Carelli 1995).

$\mathrm{Au}$ Canada, les formations audiovisuelles auprès de communautés autochtones ont été nettement plus intégrées à une politique d'État. Dans ce cadre le programme Société nouvelle, lancé en 1966 par l'Office national du film (ONF), est crucial. Il consiste à faire participer des populations à l'élaboration de films et de vidéos sur leur propre réalité, ce processus étant censé faciliter les adaptations rendues nécessaires par des changements économiques ou sociaux (Boyle 1999; Marchessault 1995a, 1995b; Roth 2005; White 2006). Ainsi,

\begin{abstract}
le but avoué de Société nouvelle était d'approfondir la démocratisation du Canada en fournissant des moyens de production médiatique aux communautés marginalisées de la Nation. Plus spécifiquement, c'était de donner une voix à ceux qui étaient perçus comme sous-représentés [ ] en leur permettant d'utiliser les films, la vidéo et la télévision par câble. L'hypothèse sousentendue [ ] était qu'en engageant les communautés ciblées dans un processus de réalisation de film, il était possible de faire un film avec eux, plutôt que sur eux (Honarpishesh 2006 : 81).
\end{abstract}

Si ce programme n'est pas spécifiquement consacré aux Premières Nations, il va mettre en place une approche qui aura son importance. Ainsi You are on Indian Land (Ransen 1969), en permettant au Mohawk Mike Mitchell de réaliser un documentaire sur la protestation de sa communauté contre les taxes à payer à la frontière canadoétats-unienne (Ginsburg 1999), a fait germer l'idée du Mohawk Travelling College. Plus au nord, le plan du gouvernement fédéral du Canada qui accompagne le lancement des satellites Anik (1971-1975) repose également sur le programme Société nouvelle et finance des tournages de films et des formations en audiovisuel auprès des communautés du Nord (Roth 2005). Cet investissement étatique s'étiole progressivement. Le programme Société nouvelle arrive à son terme en 1980 (Evans 1991), et la vidéo comme instrument de changement social perd de son attrait, la fascination technologique faisant place à une analyse forcément plus nuancée (Marchessault 1995a). Aujourd'hui cependant la question des formations d'autochtones au sein même de leurs communautés reste d'actualité, notamment au Québec.

\section{Contours d'UNe eXPÉRIENCE QUÉBÉCOISE : LE WAPIKONI MOBILE}

Depuis 2004, le Wapikoni mobile organise en effet des formations audiovisuelles destinées à de jeunes autochtones dans la province. C'est un organisme sans but lucratif (OSBL), fondé en 2003 sous forme de corporation entre la maison de production Les Beaux Jours, le Conseil de la Nation atikamekw (CNA) et l'Assemblée des Premières Nations du Québec et du Labrador (APNQL). Sa principale activité consiste à offrir, dans les réserves, des formations en audiovisuel de quatre semaines à de jeunes autochtones de 15 à 35 ans. (Le Wapikoni mobile offre également des facilités d'enregistrement musical, mais cet aspect est moins pertinent par rapport à notre article.) Les premières interventions par la vidéo ont eu lieu en 2004, dans six communautés attikameks et algonquines. Le projet n'a cessé de prendre de l'ampleur, avec treize communautés distinctes (Attikameks, Algonquins, Cris et Innus) participantes en 2009 et quatorze en 2010. En 2011, avant que la perte d'une importante subvention ne bouleverse leur programme, quinze formations étaient prévues. Au total, le Wapikoni mobile a organisé des formations dans dix-neuf communautés autochtones québécoises entre 2004 et 2010, a formé 1310 jeunes, pour une production d'environ 450 films (Wapikoni mobile 2006, 2008b, 2009b, 2011b). Ces films ont été produits dans le cadre de ce que le projet appelle des "escales». Deux unités mobiles de production ont été aménagées sur des bases d'autocaravane et de caravane. Elles sont équipées de deux stations de montage, de trois ensembles caméra et prise de son, et d'un mini-studio d'enregistrement pour les voix et les musiques. Chaque escale mobilise une équipe de quatre personnes, qui sont salariées par le Wapikoni mobile. Un intervenant jeunesse est présent pendant cinq semaines et il travaille en collaboration avec un coordonnateur autochtone local. Ces deux personnes sont chargées d'identifier les participants potentiels et de faciliter le déroulement de l'escale. La formation audiovisuelle à proprement parler est dispensée par deux cinéastesaccompagnateurs, qui sont sur place pendant quatre semaines. La formation porte sur l'aide à la conception (choix d'un sujet, écriture), le tournage (prises de vue à l'aide de caméras, prise de son, direction d'acteurs) et le montage.

Le Wapikoni mobile s'est donné pour vocation de former progressivement, à travers la pratique, des participants qui débutent souvent sans aucune notion de réalisation audiovisuelle. C'est un mélange d'apprentissage formel et de réalisation concrète, synthétisé comme " pédagogie siamoise » ou « apprendre en faisant » (Wapikoni mobile 2009d : 2), qui voit les cinéastes-accompagnateurs intervenir activement dans le processus de réalisation. Ce choix est imputable à la fois à des considérations pragmatiques qui ont trait au besoin de résultats concrets - notamment par rapport à l'activité de diffusion du projet ou aux exigences d'une intervention rapide - et à des questions d'ordre pédagogique. Le Wapikoni mobile considère ainsi qu'il serait contre-productif de confronter les participants à des situations d'échec; l'implication concrète du formateur permet de contourner ce risque. Plus fondamentalement, ce choix traduit l'objectif premier du Wapikoni mobile: avant même de former des professionnels de l'audiovisuel, il s'agit de "permettre aux jeunes de s'exprimer », de favoriser l'émergence " de nouvelles passions [...] le dépassement de soi et l'initiative artistique » (Wapikoni mobile 2009c : 2). On retrouve ici des traits du programme Société nouvelle, ce qui montre son poids dans la tradition de l'intervention par la vidéo au Canada : la prise de parole est considérée comme une vertu en soi. Cependant, ce projet ne se limite pas à cet horizon, et les formations dépassent rapidement le cadre de l'expression de soi et de la seule initiation. Le niveau des formations qui ont lieu dans le cadre des escales dépend en grande 
partie de la motivation des participants, pendant les quatre semaines de présence du Wapikoni mobile dans leur communauté, mais également à travers les années, puisque ce dernier revient quasi systématiquement dans les communautés où il a commencé à intervenir. Et, de fait, un certain nombre de jeunes autochtones y participent d'année en année et acquièrent des compétences de plus en plus poussées. On a ainsi rencontré des jeunes qui s'étaient inscrits dans des formations audiovisuelles, ou qui comptaient reprendre des études abandonnées. La possibilité de la formation courte dispensée à Wendake par l'Université du Québec à Chicoutimi (UQAC), « Programme court en production audiovisuelle des Premières Nations », a permis à plusieurs jeunes formés par le Wapikoni mobile de se perfectionner en audiovisuel. Cela dénote l'émergence ou le renforcement de réelles motivations professionnelles. De même, trois participants ont été sélectionnés dans le cadre du programme "Place aux histoires autochtones » mis en place depuis 2008 par Téléfilm Canada, en collaboration avec APTN (Wapikoni mobile 2009b). La formation dispensée lors des escales du Wapikoni mobile permet ainsi l'émergence de talents au gré des motivations personnelles, même si elle ne débouche pas systématiquement sur des niveaux de compétence suffisamment élevés pour que les participants soient autonomes. Même si ces informations restent partielles, elles dénotent toutefois une contribution du Wapikoni mobile à l'émergence d'une génération de réalisateurs et de professionnels de l'audiovisuel dont l'évolution sera intéressante à observer.

On peut relever deux différences notables entre la formation dispensée par le Wapikoni mobile et les autres projets de formation audiovisuelle documentés. Alors que les expériences aux États-Unis, au Brésil ou en Australie ont des liens souvent directs avec le domaine de l'anthropologie (Ginsburg 1995; Michaels 1994; Worth et Adair 1997), au Canada c'est l'approche documentaire qui prévaut. Cette tendance est confirmée par le Wapikoni mobile, qui est le fruit d'une mobilisation de la documentariste Manon Barbeau et qui repose sur des compétences issues du monde de l'audiovisuel, que ce soit pour la gestion courante du projet ou pour les formations. Il partage toutefois avec tous ces projets une préoccupation pour l'expression audiovisuelle de populations qui, avant leur intervention, ne possédaient pas les ressources matérielles et cognitives adéquates à la production de leurs propres contenus vidéo. Plus fondamentalement, le contexte d'intervention du Wapikoni mobile est singulier. En effet, au-delà de leurs spécificités, les projets de formation audiovisuelle de populations autochtones sont reliés à l'introduction d'une nouvelle technologie médiatique : la télévision. C'est le cas dans le Nord canadien (Roth 2005), dans le centre de l'Australie (Ginsburg 1995; Michaels 1994) ou au Brésil (Gallois et Carelli 1995; Turner 1990). Or, dans le cas du Wapikoni mobile, la situation est différente, puisque la télévision est devenue depuis longtemps un phénomène quotidien dans les réserves, même les plus éloignées, même celles qui ne sont pas reliées au réseau électrique. Nous sommes donc moins dans un phénomène de réaction à une technologie que dans une logique de réappropriation. Cela ne signifie pas que, sur le fond, les questions qui se posaient à ces communautés confrontées subitement au petit écran et à ses contenus exogènes ne se posent plus. Les questions de l'identité culturelle, de la préservation de la langue, de la construction d'une culture hybride (Ginsburg 1995; Michaels 1994; Valaskakis 1993, 2005) restent tout à fait pertinentes. Et, de fait, il serait tout à fait possible de faire une analyse des formations et des films du Wapikoni mobile avec cette grille de lecture. Elle montrerait très probablement que des phénomènes similaires sont à l'œuvre et que l'apport du Wapikoni mobile est tout à fait significatif.

Il nous semble toutefois qu'il pourrait être utile de proposer une autre grille d'analyse. Lorsqu'on lui demandait de définir un « contenu aborigène » en Australie, Eric Michaels défendait une position quelque peu iconoclaste. D'une part, il se refusait à considérer qu'il puisse exister une définition de ce genre, car les implicites d'un tel travail de catégorisation pourraient détruire entièrement les aborigènes. De l'autre, il renvoyait aux expérimentations audiovisuelles locales de communautés warlpiries, dans le centre de l'Australie, comme pourvoyeuses de tels contenus. La grande différence était qu'à ses yeux, ces médias n'étaient pas « aborigènes»; ils étaient surtout « locaux ». Leur « aboriginalité » n'était, alors, que le produit de leur diffusion auprès de communautés non aborigènes, qui les catégorisaient ainsi (Michaels 1994 : 20-47). Et c'est bien cette distinction, cette dialectique, entre une perspective locale et une perspective exogène qui nous interpelle.

\section{FORMATIONS AUDIOVISUELLES ET CONTRE-PUBLICS SUBALTERNES}

Les Premières Nations ont en effet à composer avec des représentations souvent négatives à leur endroit, que contribue à nourrir leur représentation médiatique. Augie Fleras souligne ainsi le traitement réservé aux minorités ethniques et autochtones, surreprésentées dans les domaines politiquement secondaires (crime, divertissement) et sous-représentées dans les domaines politiques ou économiques. Et lorsque ces catégories de population sont médiatisées, elles le doivent encore largement à des contextes de crise (Fleras 2009). Un constat partagé par Robert Harding, dont l'analyse de médias écrits canadiens montre que les clichés comme l'incompétence, la corruption, et généralement lincapacité des autochtones à assumer leur destinée perdurent largement (Harding 2005). La manière dont sont représentés les autochtones et dont ces représentations sont utilisées dans des conflits politiques est donc essentielle (Valaskakis 1993). Dans son analyse du conflit entre les Innus de la Côte-Nord et l'Armée de l'air canadienne à propos de zones aériennes d'exercice, Peter Armitage souligne donc logiquement l'importance des médias dans les luttes politiques des Premières Nations au Québec : ils sont cruciaux dans la conquête de l'opinion publique, sans laquelle elles ne peuvent inverser le rapport de force inégal avec l'État fédéral. Dans ce cadre, 
la maîtrise des codes dominants, qui aident à asseoir la légitimité des parties en conflit aux yeux d'une opinion publique à conquérir, s'avère cruciale. Or, comme le montre le cas étudié, cet apprentissage est complexe, long, et entraîne des coûts : en l'occurrence, la lutte a été perdue (Armitage 1992).

C'est ici que la proposition de « contre-public subalterne » de Nancy Fraser s'avère, à notre avis, utile. Elle définit ces derniers comme des

arènes discursives parallèles dans lesquelles les membres des groupes sociaux subordonnés élaborent et diffusent des contrediscours, ce qui leur permet de développer leur propre interprétation de leurs identités, de leurs intérêts et de leurs besoins. [...] D'une part, ils fonctionnent comme des espaces de repli et de regroupement; $d$ 'autre part, ils fonctionnent comme des bases et des terrains d'essai pour des activités d'agitation dirigées vers les publics plus larges (Fraser 2005 : 126-128).

Le terme de «subalterne» n'est pas forcément des plus heureux dans un contexte autochtone. Gail Valaskakis le relie ainsi à un phénomène d'exclusion (Valaskakis 1993). Mais il serait erroné de considérer ce concept comme stigmatisant per se. En effet, il est employé dans deux sens complémentaires. D'une part, il reflète une situation d'exclusion sociale réelle. De l'autre, il souligne une dynamique de repli temporaire par rapport à une arène plus large dominée par d'autres groupes sociaux. D'un point de vue gramscien, il s'agit d'espaces d'apprentissage où des groupes dominés peuvent élaborer une contre-hégémonie sans pour autant risquer des défaites qui pourraient s'avérer coûteuses. Dans cette perspective, le passage d'un niveau d'apprentissage expérimental à celui d'une pratique «locale », pour reprendre le mécanisme mis en évidence par Eric Michaels, est particulièrement important. La dynamique sous-jacente à un contre-public subalterne suppose en effet que l'ensemble d'un groupe social donné puisse participer à une dynamique médiatique relativement autonome, c'est-à-dire autocentrée. L'expérience warlpiri « Yuendumu TV » documentée par Eric Michaels pourrait répondre à ces critères (Michaels 1994). Or, dans le cadre du Wapikoni mobile, deux expériences s'en rapprochent.

\section{DES TENTATIVES D'INSTALLATION DE STRUCTURES PÉRENNES : LES STUDIOS PERMANENTS}

Si les «studios permanents» ne font pas partie du projet dès sa genèse, ils apparaissent très rapidement aux yeux des acteurs du Wapikoni mobile comme un développement nécessaire (Wapikoni mobile 2005). Ils proposent donc aux conseils de bande de s'engager financièrement dans la mise en place de structures de production audiovisuelle permanentes dans leur communauté. Le premier studio a ouvert ses portes en janvier 2006 dans la communauté de Wemotaci. La même année, la création de deux autres studios est annoncée: Mashteuiatsh en mai et Kitcisakik en juillet (Wapikoni mobile 2007). Le studio permanent de Mashteuiatsh est inauguré en 2007, mais dès 2008 le manque d'implication financière des autorités locales rend son existence difficile (Wapikoni mobile 2008b) et cette initiative est, depuis, en sommeil. Aujourd'hui les studios permanents créés dans le cadre du Wapikoni mobile à Wemotaci et à Kitcisakik sont les seuls en activité. Ces structures sont pour l'instant plus modestes que des télévisions locales en termes d'équipement et de ressources humaines, même si le studio de Wemotaci semble connaître un développement important qui pourrait rendre assez rapidement ce constat caduc (Wapikoni mobile 2009b). Les équipements consistent en quelques caméras de type PD170, qui sont à la fois de bonne qualité et relativement bon marché par rapport aux équipements professionnels, ainsi que des ensembles de micros (perche et cravate) pour les prises de son. Un ou deux ordinateurs sont destinés au montage et aux tâches administratives.

Ces studios ont fonctionné avec peu de ressources humaines permanentes et stables, notamment en raison de la difficulté à générer suffisamment de revenus. Ainsi, le studio de Kitcisakik connaît depuis sa création des hauts et des bas, en fonction de l'implication des jeunes dont le niveau de formation permet la production de contenus audiovisuels. Il ressort de quelques entretiens réalisés sur place que deux facteurs jouent un rôle particulièrement important dans cette situation. D'une part, la mise en place d'un modèle économique stable ne serait pas sans poser de difficulté dans cette petite communauté isolée de 450 personnes. S'ils ont bénéficié, tout comme le studio de Wemotaci, de multiples commandes qui émanaient du ministère de la Santé, du programme Liaison justice, d'Hydro-Québec (Wapikoni mobile 2008b), ainsi que de l'implication des conseils de bande et d'autres institutions autochtones, ces ressources financières se sont pour l'instant avérées à la fois trop ponctuelles et trop mal intégrées par les équipes de production pour soutenir la pérennisation du studio. Or il est nécessaire de pouvoir salarier des permanents afin d'assurer la pérennité du studio. D’autre part, il semblerait que les différentes institutions locales n'aient pas développé une vision d'ensemble cohérente sur la place et l'apport à la collectivité d'une unité de production audiovisuelle permanente. C'est précisément ce qui semble être en cours de développement, par contre, dans la communauté de Wemotaci. D’après les rapports annuels du Wapikoni mobile, c'est ce studio, créé le premier, qui a été le plus stable et le plus dynamique des trois, bien qu'il ait pu lui aussi connaître des hauts et des bas, toujours pour des raisons de financement. Depuis 2009 un plan de développement très ambitieux existe dans cette communauté. Le studio permanent serait rapproché de la radio locale et du journal communautaire et ferait partie d'un "pôle d'excellence » à la fois de production et de formation, où la place du multimédia serait centrale. Ce projet, ambitieux y compris d'un point de vue financier, serait activement soutenu par le conseil de bande qui aurait développé une politique de communication d'envergure, à la fois pour communiquer à l'intérieur de la communauté et vers l'extérieur.

Ces deux expériences permettent d'imaginer la structuration d'unités locales de production, mais peuvent également préfigurer la mise en place de contre-publics subalternes. En effet, elles ne sont pas limitées à des films 
de commande pour informer la population des services locaux (santé, service public). Même si cette préoccupation est importante, notamment pour justifier les investissements des conseils de bande, d'autres types de production existent. Une logique mémorielle est clairement à l'œuvre, puisque l'archivage de témoignages d'aînés, à propos par exemple de la construction traditionnelle de canots ou de savoirs médicinaux, est une préoccupation centrale depuis les premiers pas de ces studios. D'autres vidéos répondent davantage à des logiques de sensibilisation des populations locales, en termes de gestion de l'environnement notamment (pêche, foresterie...). Sur un plan plus politique, ils ont pu amener la communauté à faire l'expérience, complexe et conflictuelle, de la médiatisation d'enjeux locaux comme les élections au conseil de bande. Qui peut parler? De quel sujet? De quelle manière? Comment gérer ce mode de communication des discours publics, qui ont leur forme propre par rapport aux exigences du medium? Autant de questions centrales par rapport à la logique d'apprentissage contenue dans la proposition de contrepublic subalterne. Les studios permanents issus du Wapikoni mobile posent les premiers jalons d'un travail de la communauté sur elle-même, tant en termes de construction de son identité hybride qu'en termes d'exercice médiatico-politique.

Sans que le rôle central du Wapikoni mobile ait fondamentalement changé (il s'agit toujours en premier lieu d'offrir des activités constructives, valorisantes et formatrices à de jeunes autochtones en mettant temporairement des outils d'expression audiovisuelle dans leur réserve), on peut raisonnablement supposer qu'il contribue à l'établissement de médias audiovisuels autochtones au Québec. Peut-on parler pour autant de contre-publics subalternes? Michael Warner reproche à Nancy Fraser de rester trop dépendante d'un rapport conscient du groupe à soi-même (Warner 2002); il nous semble pourtant qu'il voudrait ainsi priver ce concept d'une composante essentielle. C'est justement parce qu'ils reposent sur une prise de conscience de leur communauté d'intérêts que ces contre-publics s'organisent. Dans le cas qui nous préoccupe, l'existence de cette prise de conscience ne se pose même pas. Il paraît, par contre, trop tôt pour vouloir analyser ces expériences à l'aune de la production de discours médiatiques à même de porter efficacement des luttes dans l'arène médiatique dominante. Il est, toutefois, sans doute possible de faire une comparaison avec une expérience plus ancienne : le développement des médias autochtones dans le Nord canadien.

\section{L'eXPÉRIENCE dANS LE NORd DU CANADA： tROIS NIVEAUX DE DÉVELOPPEMENT}

Le travail de Lorna Roth (2005) sur le développement des médias audiovisuels publics autochtones du Nord canadien peut être lu comme une modélisation possible de l'installation progressive de médias autonomes. Comme nous l'avons évoqué, le gouvernement fédéral a accompagné le lancement des satellites Anik, au début des années soixante-dix, d'un programme de formation des populations locales à la vidéo. Dans un premier temps, des expérimentations restreintes ont eu lieu dans chaque communauté, où le "procès vidéo » était plus important que le « produit vidéo »: c'est ce qui se passe pendant la réalisation de contenus audiovisuels qui est jugé réellement important, et non la diffusion des résultats obtenus (Roncagliolo 1991; Roth 2005). Ces ateliers vidéo, a priori destinés à accompagner le « développement » que devait produire, dans une approche à la fois technico-déterministe et diffusionniste très marquée par les préceptes d'Everett Rogers (Rogers 1962), l'introduction de la communication par satellite, ont ainsi contribué à faire émerger des compétences locales, et plus généralement à renforcer une prise de conscience de l'importance d'une maîtrise des médias par les populations locales, et plus spécifiquement par les responsables politiques (Roth 2005 : 105). La technologie audiovisuelle a ainsi été expérimentée et progressivement maîtrisée à une échelle locale, tant en termes de production que de diffusions. Dans un second temps, Roth note un travail de liaison entre les différentes communautés. Celles-ci développent des échanges de plus en plus structurés, jusqu'à créer la Northern Québec Inuit Association (NQIA) en 1974 (ibid. : 107). Progressivement, la volonté de créer un réseau de diffusion propre voit le jour, bien que la logique d'une production destinée à des diffusions locales prédomine (ibid. : 110). Parallèlement, on observe une structuration progressive d'unités de production audiovisuelles, les Native Communication Societies (NCS), toujours à vocation locale - elles sont subventionnées pour cela -, mais qui diffusent de plus en plus à une échelle régionale et pensent de plus en plus à diffuser vers le Sud, notamment à partir du milieu des années quatre-vingt (ibid. : 178). Cette dynamique aboutit finalement à la création d'une programmation "panNord »: Television Northern Canada (TVNC) est autorisée par le Conseil de la radiodiffusion et des télécommunications canadiennes (CRTC) en 1991. Des programmes voyagent ainsi entre les différentes communautés du Nord. Cependant, cela n'est considéré que comme une étape par les artisans de ces médias, qui considèrent qu'à terme l'objectif est de diffuser la réalité inuite aux populations du Sud (ibid. : 187, 198). Cela se concrétise par le lancement d'APTN sur l'ensemble du Canada (1997-1999). Ce réseau de diffusion fait partie du service de base obligatoirement fourni par les câblo-opérateurs et diffuseurs satellites canadiens, qui doivent de plus le rémunérer à hauteur de 0,15 \$ par abonné. Roth y voit l'affirmation pour les Premières Nations qu'elles sont « one of the three Founding Nations of Canada », au même titre que les Français et les Anglais (Roth 2005 : 204).

Cette structuration progressive est très dépendante d'un contexte historique et politique particulier. Ainsi, l'histoire de la Central Australian Aboriginal Media Association (CAAMA) montre que ce rythme peut changer. Structurée initialement, et avec succès, autour de la radio, la CAAMA s'est retrouvée en position d'assumer une offre télévisuelle par satellite sans avoir réellement eu le temps de profiter de la mise en synergie d'expériences locales, qui débutaient presque en même temps (Ginsburg 1995; 
Michaels 1994). Il n'est pas dit non plus que le lent cheminement qui a abouti à la création d'APTN soit garant d'une meilleure relation avec les groupes de production audiovisuelle locaux, ou de choix techniques et éditoriaux différents. Il est frappant en effet de voir à quel point les critiques adressées à Imparja, l'équivalent australien d'APTN, sont similaires à celles faites à l'encontre de celle-ci aujourd'hui (George et Aubi, dans ce numéro; Michaels 1994). Cependant, les trois niveaux complémentaires dans l'ancrage de ces pratiques audiovisuelles mis en évidence par Lorna Roth restent, à notre avis, valables, y compris dans le cas australien. Premièrement, il existe une appropriation locale des technologies audiovisuelles qui laisse la place à l'expérimentation non seulement des techniques, mais également des pratiques sociales. Deuxièmement, on voit des échanges de plus en plus structurés entre communautés, et la naissance d'un outil de représentation médiatique commun où les différentes réalités peuvent être comparées. Troisièmement, une nouvelle dynamique de communication se met en place, orientée non vers un public autochtone, mais vers le reste de la population du Canada.

Pour reprendre la grille d'analyse empruntée à Nancy Fraser, on peut considérer que les deux premiers niveaux participent de " contre-publics subalternes », tandis que le troisième utilise les compétences acquises pour entrer de plain-pied dans la lutte pour la définition des représentations. Ainsi, Roth souligne que, si les acteurs des NCS partageaient la certitude qu'il est nécessaire de diffuser vers les allochtones, ils étaient également d'accord pour considérer qu'il était nécessaire, dans un premier temps, d'établir un service en langue autochtone à destination des communautés autochtones, de manières transversale et pérenne (Roth 2005 : 183). Ailleurs, Roth note que les chefs autochtones ont dû apprendre à maîtriser le langage médiatique, y compris dans le cadre de diffusions audiovisuelles entre communautés, en parlant en phrases courtes et en s'appuyant sur la rhétorique technicorationnelle dont Armitage avait relevé l'importance (Armitage 1992). En d'autres termes, les expérimentations essentiellement techniques des débuts du programme d'appui aux médias audiovisuels publics autochtones du Nord ont donné naissance à des pratiques médiatiques locales où ont été expérimentés représentation de soi, débat, désaccords, le tout dans une situation de repli effectif puisqu'elles étaient limitées essentiellement aux communautés de production. Ce n'est que dans un second temps que ces productions ont pu donner lieu à des échanges entre communautés autochtones et qu'elles ont contribué à l'émergence d'une prise de conscience accrue posée par la communication publique et ses enjeux pour les autochtones. Ainsi, lorsque Roth souligne l'apparition de stratégies médiatiques qui font appel à des tactiques « de hontes » et « de l'embarras» (Roth 2005 : 183-184), cela indique que les responsables politiques ont été en mesure de développer une connaissance et une maîtrise de stratégies médiatiques destinées à être utilisées dans leurs luttes politiques, notamment avec le gouvernement fédéral canadien. Il semble donc que les expérimentations locales aient permis une appropriation graduelle d'une technologie médiatique, tout comme le conceptualise Nancy Fraser, en commençant par une maturation dans des arènes relativement autonomes, peu intégrées dans l'arène médiatique canadienne, pour déboucher in fine sur une politique communicationnelle concertée destinée en priorité à un public allochtone, à qui il est nécessaire de montrer des réalités peu ou mal connues et envers qui il est nécessaire de s'affirmer.

Par rapport au développement des médias audiovisuels publics autochtones du Grand Nord canadien, les studios permanents issus de la dynamique engendrée par le Wapikoni mobile évoquent évidemment la mise en place de structures de production autonomes locales et endogènes comme les Native Communication Societies. Une étude spécifique et approfondie de ces dynamiques reste nécessaire, et il est par ailleurs sans doute trop tôt pour proposer une analyse cohérente de leur mise en place, car elles sont encore dans une phase de structuration. On peut toutefois comprendre que le Wapikoni mobile a joué et joue encore un rôle crucial dans la mise en place de studios permanents. Ce sont évidemment ses interventions dans le cadre des escales, ainsi que la mise à disposition de caméras, d'ordinateurs, et les offres de formation qui ont, dans un premier temps, fait émerger des intérêts pour l'audiovisuel chez certains jeunes et dans certaines communautés. Lidée de studios permanents doit ainsi beaucoup à la motivation de jeunes participants née lors des escales (Wapikoni mobile 2007), entre autres pour pouvoir aborder de manière plus approfondie des aspects techniques comme le montage. La création des studios permanents a donc abouti à des formations complémentaires par rapport aux aspects abordés lors des ateliers d'escale. Il est possible que le Wapikoni mobile ait renforcé certains désirs latents, tout comme il est possible qu'il ait ouvert des portes sur des possibilités jusqu'alors insoupçonnées. Mais il est certain que l'intervention du Wapikoni mobile a joué un rôle essentiel dans l'émergence puis dans la structuration de ces embryons de médias audiovisuels autochtones.

\section{Conclusion}

Dans l'état actuel des choses, on peut dire que ces studios permanents participent avant tout d'une dynamique qui relève du premier niveau identifié à partir du travail de Roth, celui de l'expérimentation locale et uniquement locale. Toutefois, le second, qui se traduit par des échanges médiatiques formels entre plusieurs communautés autochtones et par la création de facto d'une arène médiatique qui leur est propre, commence à être perceptible. Dans une large mesure, les studios permanents ont été des lieux d'expérimentation avant tout dans les communautés où ils sont implantés. Cependant, certaines commandes de film et développements institutionnels montrent qu'il existe déjà des pistes de formalisations de ce type d'échange. Ainsi, lorsque le Conseil en éducation des Premières Nations passe des commandes de film aux thématiques 
similaires à tous les studios permanents, avec l'intention de pouvoir les faire circuler entre les communautés et ainsi faire la preuve de problématiques communes, cela montre qu'il existe un intérêt transversal des communautés autochtones au Québec pour ce genre d'initiative. Ces quelques éléments permettent de voir qu'il existe un mouvement similaire à celui relevé par Lorna Roth à propos de l'évolution du programme Société nouvelle, qui était progressivement passé d'une logique d'animation par l'audiovisuel à celle de la mise en place de médias communautaires autochtones. Comme dans le Grand Nord canadien, cette activité de formation permet à des individus des Premières Nations du Québec de s'initier à l'audiovisuel, voire de développer peu à peu des compétences précises. On retrouve un peu la logique de l'animation notée par Roth : le but des ateliers dispensés dans les escales n'est en effet pas nécessairement de former des professionnels de l'audiovisuel. Il s'agit en priorité de fournir une activité structurante à une jeunesse en situation précaire, de lui donner une occasion de s'exprimer - et de la valoriser. Mais, comme une proportion non négligeable de jeunes participe chaque année aux ateliers, la formation dispensée lors des escales du Wapikoni mobile permet l'émergence de talents au gré des motivations personnelles. Outre le parcours de certains individus, les studios permanents en sont sans doute la meilleure traduction. À travers ceux-ci, on peut considérer que l'activité du Wapikoni mobile a des retombées par rapport au second niveau d'appropriation des technologies audiovisuelles identifié à la suite de Roth et, par conséquent, dans la structuration d'une arène médiatique audiovisuelle propre aux différentes communautés autochtones du Québec. C'est évidemment le troisième niveau, celui qui consiste à sortir de la position de repli propre au contre-public subalterne pour entrer de plain-pied dans une arène médiatique dont les logiques, les langages, les enjeux ont peu à peu été suffisamment maîtrisés pour pouvoir rivaliser avec les acteurs sociaux en place, qui est le plus lointain. Sur ce plan, faire la part des choses dans le cas du Wapikoni mobile est complexe. Son effort soutenu de diffusion au sein de l'arène médiatique est évidemment à prendre en considération lorsqu'il s'agit de penser dans sa globalité la place des contenus autochtones au sein de l'arène médiatique québécoise. Mais si l'on prend pour cadre d'analyse la formalisation progressive de médias audiovisuels publics autochtones, il faut reconnaître que son mode opératoire limite pour l'instant la portée de son apport à ce niveau. Il pourrait cependant difficilement en être autrement. Le développement d'un réseau d'échanges de contenus audiovisuels, qui accompagne des débats entre les différentes communautés et nourrit un contre-public subalterne, comme cela a pu être le cas dans le Grand Nord, suppose non seulement un niveau d'activités plus important que celui que peut assumer à lui seul le Wapikoni mobile, qui en fait déjà beaucoup, mais également une prise en main par les communautés elles-mêmes, et notamment par les autorités locales, de la question de la communication audiovisuelle. Cependant, par plusieurs aspects, l'activité du Wapikoni mobile semble à même de constituer un terrain favorable pour ce type de développement. Il montre également qu'une analyse de ce type de formation à partir de la théorie des contre-publics peut compléter celles qui ont insisté, à juste titre, sur le travail de construction d'une identité autochtone contemporaine (Ginsburg 1995; Gallois et Carelli 1995; Michaels 1994; Valaskakis 2005). Car, si un espace public « subalterne » peut s'avérer pertinent dans une dynamique où les communautés doivent s'engager dans un travail de sauvegarde de leurs spécificités culturelles dans un monde globalisé, il ne faut pas nécessairement le concevoir comme un mécanisme uniquement défensif. Qu'une dynamique structurée autour de l'audiovisuel puisse contribuer à réaffirmer et réinventer, pour les membres de communautés autochtones, leur culture propre est, en soi, un apport inestimable. Mais elle reste le fruit d'une adaptation à des colonisations de leur monde vécu par des tendances qui, elles, restent inchangées. À moins qu'elles ne soient en retour remises en question, en utilisant les mêmes techniques et les mêmes médiums.

\section{Médiagraphie}

ARMITAGE, Peter, 1992 : « Les premières nations, les médias et le pouvoir de l'opinion publique». Anthropologie et sociétés 16(3) : 77-101.

BOYLE, Deirdre, 1999 : «O, Canada! George Stoney's Challenge ». Wide Angle 21(2) : 48-59.

EVANS, Gary, 1991: In the national interest: a chronicle of the National Film Board of Canada from 1949 to 1989. University of Toronto Press, Toronto.

FLERAS, Augie, 2009 : «Ethnic and Aboriginal Media in Canada: Crossing Borders, Constructing Buffers, Creating Bonds, Building Bridges », in Rainer Geissler et Horst Pöttker (dir.), Media, migration, integration: European and North American perspectives : 143-179. Bielefeld, New Brunswick, New Jersey.

FRASER, Nancy, 2005 : Qu'est-ce que la justice sociale? Reconnaissance et redistribution. La Découverte, Paris.

GALLOIS, Dominique T., et Vincent CARELLI, 1995 : «Video e dialogo cultural - Experiencia do projecto video nas aldeias ». Horizontes Antropologicos 1(2) : 61-72.

GINSBURG, Faye, 1995 : « Mediating Culture. Indigenous Media, Ethnographic Film, and the Production of Identity », in Leslie Devereaux et Roger Hilman (dir.), Fields of Vision: Essays in Film Studies, Visual Anthropology and Photography: 256-291. California University Press, Berkeley.

—, 1999 : «The After-Life of a Documentary: The Impact of You Are on Indian Land». Wide Angle 21(2) : 60-67.

HARDING, Robert, 2005 : "The media, aboriginal people and common sense ». The Canadian Journal of Native Studies 25(1): 311-335.

HONARPISHESH, Farbod, 2006 : «You are on Indian land », in Jerry White (dir.), The Cinema of Canada: 81-89. Wallflower Press, Londres.

MARCHESSAULT, Janine, 1995a: "Amateur Video and the Challenge for Change », in Janine Marchessault (dir.), Mirror Machine: Video in the Age of Identity: 13-25. YYZ Books \& CRCCII, Toronto.

- 1995b: "Reflections on the Dispossessed». Screen 36(2) : 131-146.

MICHAELS, Eric, 1994: Bad Aboriginal Art. Tradition, Media, and Technological Horizons. University of Minnesota Press, Minneapolis. 
RANSEN, Mort, 1969 : You Are on Indian Land. Film disponible sur DVD, 36 min, 48 s. National Film Board / Office national du film, Montréal.

RODRIGUEZ, Clemencia, 2001 : Fissures in the Mediascape: An International Study of Citizens' Media. Hampton Press, Cresskill, New Jersey.

ROGERS, Everett, 1962 : Diffusion of Innovations. The Free Press, New York.

RONCAGLIOLO, Rafael, 1991 : « The Growth of the Audiovisual Imagescape in Latin America », in Alain Ambrosi et Nancy Thede (dir.), Video, the Changing World: 22-30. Black Rose Books, Montréal/New York.

ROTH, Lorna, 2005 : Something New in the Air: The Story of First Peoples Television Broadcasting in Canada. McGill-Queen's University Press, Montréal, Kingston, Londres.

TURNER, Terence S., 1990: «The Kayapo Video Project: A Progress Report». Commission on Visual Anthropology Review (Autumn): 7-10.

VALASKAKIS, Gail Guthrie, 1993 : «Parallel Voices: Indian and Others - Narratives of Cultural Struggle ». Canadian Journal of Communication 18(3). Disponible sur Internet : <http://cjconline.ca/index.php/journal/article/view/756/662>, (consulté le 14 décembre 2011).
—, 2005 : Indian Country: Essays on Contemporary Native Culture. Wilfrid-Laurier Press, Waterloo.

WAPIKONI MOBILE, 2005: Rapport annuel 2004. Wapikoni mobile, Montréal.

—, 2006 : Rapport annuel 2005. Wapikoni mobile, Montréal.

—, 2007 : Rapport annuel 2006. Wapikoni mobile, Montréal.

—, 2008a : Rapport annuel 2007. Wapikoni mobile, Montréal.

—, 2008b : Rapport annuel 2008. Wapikoni mobile, Montréal.

—, 2009a : « Plaquette d'information ». Wapikoni mobile, Montréal.

—, 2009b : Rapport annuel 2009. Wapikoni mobile, Montréal.

—, 2009c : «Trousse d'escale ».Wapikoni mobile, Montréal.

_, 2011a: «Calendrier prévisionnel des escales». Wapikoni mobile, Montréal.

—, 2011b. : «L'univers Wapikoni ». Voir <http://wapikoni.tv/ univers/about/>, (consulté le 29 mars 2011).

WARNER, Michael, 2002 : «Publics and Counterpublics ». Public Culture 14(1): 49-90.

WHITE, Jerry, 2006 : « The Winds of Fogo », in Jerry White (dir.), The cinema of Canada: 73-79. Wallflower Press, Londres.

WORTH, Sol, et John ADAIR, 1997: Though Navajo Eyes. University of Mexico Press, Albuquerque.
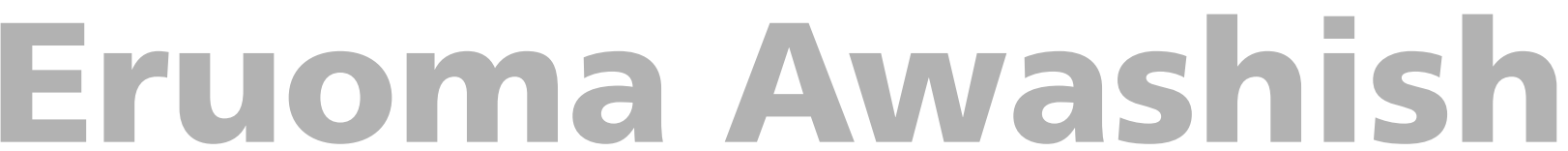

\section{Dans un miroir, un combat Acrylique et feuilles d'or sur toile, 2013 $91,4 \times 60,9 \mathrm{~cm}$ (Photo Eruoma Awashish)}

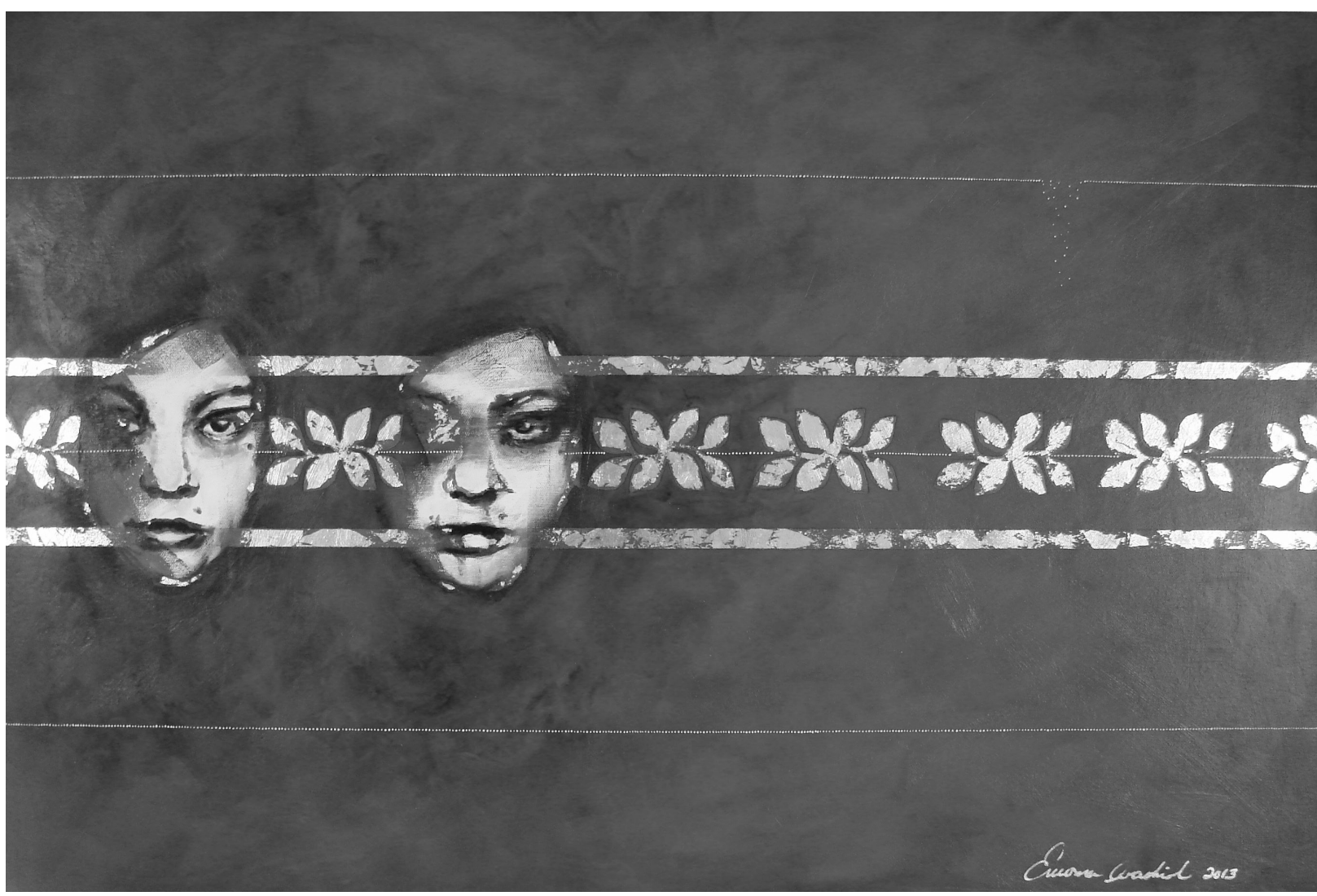

